\title{
Leptin, nutrition, and the thyroid: the why, the wherefore, and the wiring
}

\author{
Jeffrey S. Flier, Mark Harris, and Anthony N. Hollenberg \\ Division of Endocrinology, Beth Israel Deaconess Medical Center, Boston, Massachusetts 02215, USA \\ Address correspondence to: Jeffrey S. Flier, Division of Endocrinology, Beth Israel Deaconess Medical Center, \\ 330 Brookline Avenue, Boston, Massachusetts 02215, USA. \\ Phone: (617)667-2151; Fax: (617) 667-2927; E-mail: jflier@caregroup.harvard.edu.
}

The function of the thyroid gland is to produce the thyroid hormones $\mathrm{T} 3$ and $\mathrm{T} 4$, which regulate gene transcription throughout the body (1). In medical practice, the thyroid becomes an issue when its size or shape becomes abnormal or when it produces too much or too little hormone. Thus, we typically think of the thyroid with reference to the clinical states of goiter, or hyper- or hypothyroidism. But what is the physiology of the thyroid when the gland and the entire hypothalamic-pituitary-thyroid axis are intact? As first year medical students ask each year, Why exactly do we have a thyroid, at all?

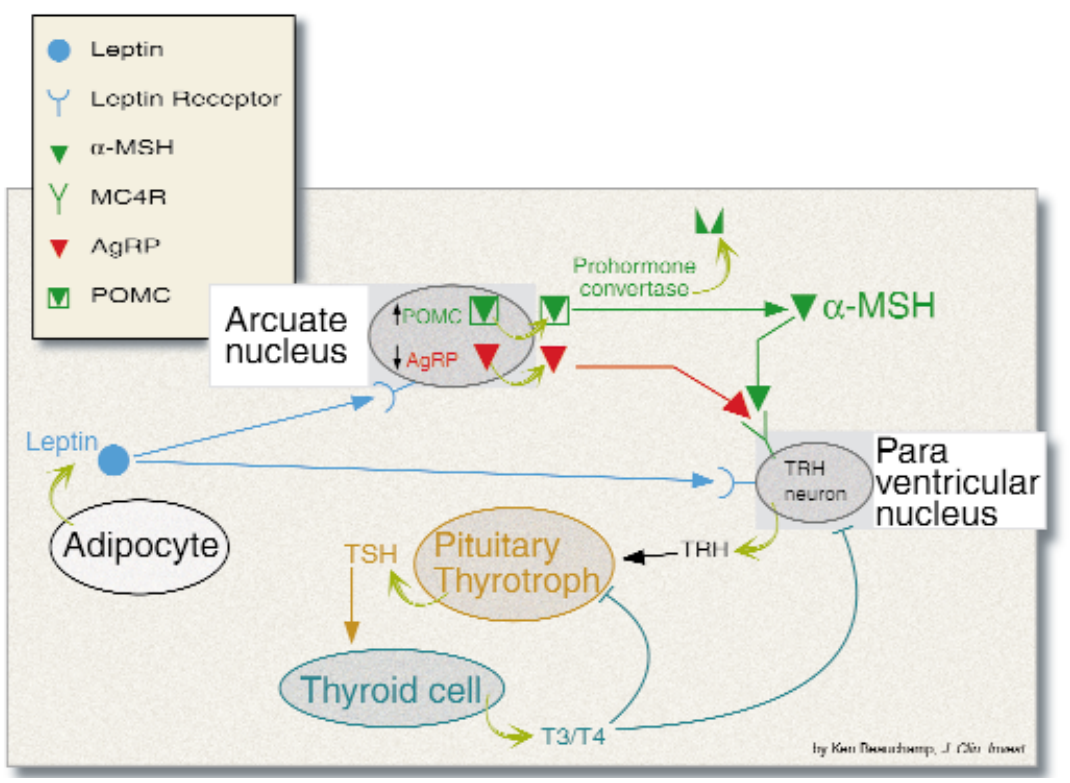

Figure 1

Maintenance of the thyroid axis by leptin through actions on the TRH neuron in the PVN of the hypothalamus. A sufficient level of leptin signaling is needed to maintain TRH expression in the hypothalamic PVN, which is necessary for normal production of TSH and production of thyroid hormones by the thyroid. Two mechanisms may be involved. In one, leptin regulates arcuate neurons expressing proopiomelanocortin (POMC) (induced by leptin) and AgRP (suppressed by leptin). These arcuate neurons project to TRH neurons, where they influence TRH expression by antagonistic actions of $\alpha-\mathrm{MSH}$ (stimulatory) and AgRP (inhibitory) on MC4Rs. Leptin may also act directly on TRH neurons through leptin receptors on these cells. In the absence of leptin signaling, the feedback loop between $\mathrm{T} 4 / \mathrm{T} 3$ and the hypothalamus-pituitary-thyroid system is lost. Hence, although levels of T4/T3 may be low, TRH and TSH levels remain suppressed.

The usual presentation of thyroid physiology does not stress dynamic changes in hormone levels. Unlike insulin or cortisol levels, which are understood to fluctuate in respectively, thyroid hormones ically thought to be maintained at a basal level of hormone that keeps the metabolic machinery humming at the proper rate. In our opinion, this static view of thyroid physiology is mistaken. occur changes in thyroid hormone that mammals, thyroid hormone levels are subject to major physiologic regulation ogy of the thyroid gland.

The thyroid system is regulated at multiple levels, one or more of which might account for nutritional adaptation. First, thyrotropin-releasing hormone, a neuropeptide produced in the paraventricular nucleus (PVN) of the hypothalamus, controls the release of thyroid-stimulating hormone (TSH) from the pituitary. TSH acts on receptors on the thyroid to promote synthesis and release of the thyroid hormones T4 and T3. In addition, a family of deiodinases metabolize the less-active T4 to the more-active $\mathrm{T} 3$ or to the inactive reverse $\mathrm{T} 3$. In primary hypothyroidism, when T3 and T4 levels fall because of a defect within the thyroid, a 2-part compensatory system kicks in. In the PVN of the hypothalamus, TRH expression increases because of the lack of negative feedback by thyroid hormones (4). In the pituitary thyrotroph, TSH production increases due to both increased TRH production and decreased negative feedback by thyroid hormones on the genes encoding TSH subunits (5). The increased TSH serves to drive the failing thyroid and is the most sensitive test for the diagnosis of thyroid failure. 
Starvation appears to act, at least in part, by suppressing TRH expression in the PVN-although, interestingly, TRH continues to be expressed in the remainder of the central nervous system (CNS), which plays no role in regulating pituitary TSH production (2). $\mathrm{TSH}$ production falls, and simultaneously the pattern of glycosylation on newly synthesized TSH is altered so that the TSH that is produced is of reduced bioactivity (6). Thus, as a consequence of starvation, T4 and T3 levels fall, leading to central hypothyroidism. Leptin and the regulation of thyroid function by the brain. The mechanism by which the brain orchestrates this adaptation is now becoming increasingly clear. The dominant, and perhaps sufficient, signal to the brain that suppresses TRH expression in the PVN is a drop in the level of the hormone leptin. This $16-\mathrm{kD}$ hormone is expressed predominantly in adipose cells, and its absence, as in the $o b / o b$ mouse, produces severe obesity (7). Initially viewed primarily as a hormone designed to prevent obesity, a substantial body of work now suggests that leptin also signals the switch from the fed to the starved state $(3,8)$. A fall in leptin acts through the hypothalamus to increase appetite, decrease energy expenditure, and modify neuroendocrine function in a direction that favors survival. The consequences of falling leptin include suppression of reproduction, linear growth, and the thyroid axis, as well as activation of the stress axis (3). As with the mouse gene, mutation of the human leptin receptor gene can also cause obesity with central hypogonadism and hypothyroidism (9).

As reviewed in ref. 10, much effort is now directed to understanding the precise neural circuits through which leptin brings about these effects on appetite and neuroendocrine function. With regard to thyroid activity, a crucial question is whether falling leptin levels are sensed directly by the leptin receptor (the ObRb isoform; ref. 11) found in TRH neurons, or indirectly, through one or more distinct leptin-responsive neurons that communicate with the TRH neuron. A recent study suggested that an indirect pathway might exist. Legradi et al. (12) chemically ablated the arcuate nucleus in rats and observed that starvation failed to suppress thyroid levels. Because the treatment they used, neonatal administration of monosodium glutamate, leaves the TRH neurons of the PVN intact, this finding suggests that leptin regulates input from the arcuate to the TRH neurons in the PVN (12).

The paper by Kim et al. in this issue of the JCI adds chemical specificity to this model (13). These authors have used in vivo and in vitro approaches to reveal a role for the melanocortin pathway in mediating the nutritional response of the TRH neuron to leptin. The melanocortin pathway involves 2 ligands expressed in distinct neural populations in the arcuate nucleus of the hypothalamus, as well as 1 receptor on which these ligands converge, antagonizing each other's effects. The ligands are AgRP, which is suppressed by leptin, and $\alpha-\mathrm{MSH}$, which is induced by leptin. The melanocortin 4 receptor (MC4R) is stimulated by the latter but inhibited by the former $(14,15)$, so the pathway can be antagonized by decreasing the agonist $\alpha-\mathrm{MSH}$, by increasing the antagonist AgRP, or by a loss in receptor function. Indeed, each of these events causes obesity $(16,17)$. The data reported here strongly suggest that the melanocortin pathway plays an important role in the regulation of the thyroid axis by leptin as well, perhaps by promoting contacts between functionally antagonistic leptin-regulated neurons in the arcuate nucleus and TRH neurons in the PVN. Kim et al (13). demonstrate that $\alpha-\mathrm{MSH}$ increases TSH levels when it is administered centrally to living rats and that it stimulates TRH release when added to hypothalamic slices. Furthermore, AgRP blocks release of TRH by antagonizing $\alpha-\mathrm{MSH}$ and thereby opposing the action of leptin. A recent report identified $\alpha-\mathrm{MSH}$ in nerve terminals innervating TRH neurons in the PVN and demonstrated that this hormone prevents the fasting-induced suppression of Pro-TRH gene expression (18). Thus, the central melanocortin system can regulate the thyroid axis and is well positioned to mediate the actions of leptin on the thyroid axis.

Many endocrine pathways are subject to regulation at several levels, and the suppression of thyroid function in starvation appears to be no exception. Preliminary data suggest that TRH neurons are direct targets of leptin as well, because leptin activates TRH promoter constructs in transfected cells (19). Further studies using different approaches will be needed to examine this question. However, it should be noted that $A^{y}$ mice, which ectopically overexpress the agouti protein, a homologue of AgRP that also antagonizes MC4R signaling, do not have hypothyroidism. This might suggest that the acute and chronic melanocortin blockade affect thyroid function differently. In any event, the precise role of the melanocortin pathway in control of the thyroid axis under a variety of physiologic and pathophysiologic circumstances remains to be determined.

Whatever the precise wiring mechanism by which it regulates the TRH neuron, leptin's ability to orchestrate changes in the thyroid axis in rodents during the transition from the fed to the starved states is established, and this nutritional response should probably be viewed as a key but underappreciated evolutionary function of the thyroid hormone system. Starvation is less immediately threatening for humans than for mice, because humans have greater energy stores compared with their metabolic rate. Accordingly, suppression of the thyroid axis during starvation in humans occurs more slowly and is of smaller magnitude. Studies of the possible role of leptin and the central melanocortin system in regulation of the hypothalamicpituitary-thyroid axis in humans are in their infancy. It is unknown, for example, whether the leptin and melanocortin pathways regulate the thyroid axis under physiologic states other than from starvation or in response to severe illness, another state in which the thyroid axis may be severely suppressed (20). As leptin and reagents that modify the melanocortin system become available for clinical research studies, many new insights are sure to emerge.

1. Hollenberg, A.N. 1998. Thyroid hormone receptor isoforms, nuclear corepressors and coactivators and their role in thyroid hormone action. Current Opinion in Endocrinology and Diabetes. 5:314-320.

2. Blake, N.G., Eckland, D.J., Foster, O.J., and Lightman, S.L. 1991. Inhibition of hypothalamic thyrotropin-releasing hormone messenger ribonucleic acid during food deprivation. Endocrinology. 129:2714-2718.

3. Ahima, R.S., et al. 1996. Role of leptin in the neuroendocrine response to fasting. Nature. 382:250-252.

4. Segerson, T.P., et al. 1987. Thyroid hormone regulates TRH biosynthesis in the paraventricular nucleus of the rat hypothalamus. Science. 238:78-80.

5. Shupnik, M.A., Chin, W.W., Habener, J.F., and Ridgway, E.C. 1985. Transcriptional regulation of the thyrotropin subunit genes by thyroid hormone. J. Biol. Chem. 260:2900-2903.

6. Weintraub, B.D., Gesundheit, N., Taylor, T., and Gyves, P.W. 1989. Effect of TRH on TSH glycosy- 
lation and biological action. Ann. NY Acad. Sci. 553:205-213.

7. Zhang, Y., et al. 1994. Positional cloning of the mouse obese gene and its human homologue [erratum 1995, 30:479]. Nature. 372:425-432.

8. Flier, J.S. 1998. Clinical review 94: what's in a name? In search of leptin's physiologic role. J. Clin. Endocrinol. Metab. 83:1407-1413.

9. Clement, K., et al. 1998. A mutation in the human leptin receptor gene causes obesity and pituitary dysfunction. Nature. 392:398-401.

10. Elmquist, J.K., Elias, C.F., and Saper, C.B. 1999. From lesions to leptin: hypothalamic control of food intake and body weight. Newron. 22:221-232.

11. Tartaglia, L.A., et al. 1995. Identification and expression cloning of a leptin receptor, OB-R. Cell. 83:1263-1271.
12. Legradi, G., et al. 1998. Arcuate nucleus ablation prevents fasting-induced suppression of ProTRH mRNA in the hypothalamic paraventricular nucleus. Neuroendocrinology. 68:89-97.

$13 \mathrm{Kim}$, M.S. et al. The central melanocortin system affects the hypothalamo-pituitary thyroid axis and may mediate the effect of leptin. J. Clin. Invest. 105:1005-1011.

14. Shutter, J.R., et al. 1997. Hypothalamic expression of ART, a novel gene related to agouti, is up- regulated in obese and diabetic mutant mice. Genes Dev. 11:593-602.

15. Ollmann, M.M., et al. 1997. Antagonism of central melanocortin receptors in vitro and in vivo by agouti-related protein [erratum, 1998 , 281:1615]. Science. 278:135-138

16. Fan, W., Boston, B.A., Kesterson, R.A., Hruby, V.J., and Cone, R.D. 1997. Role of melanocortinergic neurons in feeding and the agouti obesity syndrome Nature. 385:165-168.

17. Huszar, D., et al. 1997. Targeted disruption of the melanocortin-4 receptor results in obesity in mice. Cell. 88:131-141.

18. Fekete, C., et al. 2000. (alpha)-Melanocytestimulating-hormone is contained in nerve terminals innervating thyrotropin-releasing hormone synthesizing neurons in the hypothalamic paraventricular nucleus and prevents fasting induced suppression of Prothyrotropin-releasing hormone gene expression. J. Neurosci. 20:1550-1558.

19. Nillni, E., et al. 1999. Leptin regulates TRH biosynthesis. Endocrine Society Abstract. 36:1. (Abstr.)

20. Nicoloff, J.T., and LoPresti, J.S. 1996. Nonthyroidal illness. In The thyroid. L.E. Braverman and R.D. Utiger, editors. LippincottRaven. Philadelphia, PA. 286-296. 